\title{
Aging and contextual binding: Modeling recency and lag recency effects with the temporal context model
}

\author{
MARC W. HOWARD \\ Syracuse University, Syracuse, New York \\ MICHAEL J. KAHANA \\ University of Pennsylvania, Philadelphia, Pennsylvania \\ and \\ ARTHUR WINGFIELD \\ Brandeis University, Waltham, Massachusetts
}

\begin{abstract}
Normal aging has been shown to spare recency effects in the initiation of free recall while disrupting temporally defined associations. The temporal context model (TCM) explains recency and temporally defined associations as consequences of a gradually changing context signal and recovery of those contextual states, respectively. Here we extend TCM to account for the dissociation between recency and temporally defined associations in younger and older adults. Modeling results suggested that the effect of aging was restricted to a decrement in the ability of items to recover the temporal contexts in which they were presented, a function that has been hypothesized to depend on the hippocampus.
\end{abstract}

The temporal context model (TCM; see Howard, Fotedar, Datey, \& Hasselmo, 2005; Howard \& Kahana, 2002) offers an explanation for two basic properties of episodic memory - the recency effect and temporally defined associative effects. The recency effect is the finding that the more recent an experience is, the better it will be remembered. In TCM, the optimal cue for an item is the context in which it was encoded. Because the current state of context changes gradually over time, the present state of context overlaps more strongly with recent contexts, leading to a recency effect. This account builds on prior work that attributed temporal changes in memory to a variable context signal (Glenberg \& Swanson, 1986; Mensink \& Raaijmakers, 1988; Murdock, 1997). By postulating that the change in the contextual state from moment to moment is not random but includes contexts recovered from prior experiences with the item being presented, TCM is able to describe temporally defined associations between items presented nearby in a list (Kahana, 1996).

Recently, Howard et al. (2005) have proposed a mapping hypothesis that identifies processes in TCM with the function of different regions of the human medial temporal lobe. They argued that the current state of context should be

This research was funded by NIH Grants AG15852, MH55687, and MH069938, and by the W. M. Keck foundation. We thank Emily Dolan for helpful comments on a previous version of the manuscript. Correspondence should be addressed to M. Howard, Syracuse University, Department of Psychology, 430 Huntington Hall, Syracuse, NY 132442340 (e-mail: marc@memory.syr.edu). maintained by the entorhinal cortex, a cortical region that is reciprocally connected to the hippocampus. Howard et al. proposed that the hippocampus is responsible for binding items to the contexts in which they are presented. In this way, Howard et al. were able to account for a variety of results from lesion studies and neurophysiological recordings.

In this paper, we use aging as a neuropsychological dissociation to evaluate quantitative predictions of TCM in the free recall task. Naveh-Benjamin and colleagues (NavehBenjamin, 2000; Naveh-Benjamin, Hussain, Guez, \& Bar-On, 2003) have argued that the memory deficit in episodic tasks shown by older adults is a consequence of a failure to bind items together with their episodic contexts into a coherent whole. This associative deficit hypothesis is very similar to the item-context binding function ascribed to the hippocampus by Howard et al. (2005). In addition to well-known differences in prefrontal anatomy that occur with age (Head et al., 2004; Raz et al., 1997; West, 1996), there is ample evidence that normal aging is associated with changes in the functioning of the medial temporal lobe in general and the hippocampus in particular (see Rosenzweig \& Barnes, 2003, for a review). If the hippocampus is indeed responsible for binding items to their episodic context, then the associative deficit observed for older adults in the free recall task should follow a particular form predicted by a decrease in the recovery of temporal context in TCM. This paper tests these predictions. We start by describing changes in recency and temporally defined associations in older adults. We then provide a brief summary of TCM, with an emphasis on how it generates temporally defined associations. 


\section{Recency and Lag Recency in Normal Aging}

In free recall, participants recall as many items as they can remember from a recently presented list, in any order. If tested immediately, participants typically initiate recall by recalling a word from the end of the list, resulting in a recency effect. In free recall, the probability of first recall (PFR), a serial position curve for the first response that participants make (Hogan, 1975; Laming, 1999), is a robust marker of the recency effect. The primacy effect in free recall is generally much smaller than the recency effect and, unlike recency, depends critically on rehearsal (Brodie \& Murdock, 1977; Rundus, 1971; Tan \& Ward, 2000).

As recall progresses after the first recall, participants recall items in the order in which they come to mind. Analysis of recall order reveals a tendency to make transitions among nearby list items. The conditional response probability as a function of lag (lag-CRP; see Howard \& Kahana, 1999; Kahana, 1996) illustrates the probability that an item from serial position $i+$ lag is recalled immediately following an item from serial position $i$. The lag-CRP has a characteristic form, with recall of an item generally followed by recall of nearby items with a forward bias (see Figure 1A). We refer to this phenomenon, illustrating the effect of temporal contiguity on retrieval transitions, as the lag recency effect (Howard \& Kahana, 1999). According to TCM, the lag recency effect reflects associations formed between items presented in similar episodic contexts.

Kahana, Howard, Zaromb, and Wingfield (2002) found no difference between PFR curves from older (ages 66-88) and younger (ages 18-21) participants. However, the lag recency effect for older adults was significantly reduced, suggesting a deficit in associative processes that was consistent with Naveh-Benjamin's (2000; Naveh-Benjamin et al., 2003) associative deficit hypothesis of cognitive aging. Dual-store models of episodic recall (Atkinson \& Shiffrin, 1968; Raaijmakers \& Shiffrin, 1980) offer a straightforward account for both recency and lag recency effects. On an immediate test, recent items are present in the short-term store (STS) and can be easily recalled at the time of test. Lag recency arises because items from nearby list positions tend to occur together in STS and then become associated in the long-term store (LTS). Dissociations between recency and lag recency could thus be seen as dissociations between short- and long-term memory processes.

\section{The Temporal Context Model}

Here we provide a concise description of TCM. More detailed descriptions can be found elsewhere (e.g., Howard et al., 2005; Howard \& Kahana, 2002). In TCM, temporal context is a vector that changes gradually over time. Items, represented as vectors in semantic memory, become bidirectionally associated with context. States of context cue recall of items to the extent that they overlap with those items' encoding contexts. Changes in temporal context from one moment to the next are caused by the specific items presented rather than by random fluctuations. In particular, repetition of an item in semantic memory can recover the states of temporal context previously associated with that item. Associative effects in memory can arise because the retrieved contextual states overlap with the encoding context of nearby items. That is, associations observed between items are mediated by the effects that the items have on that current state of context.

In TCM, $\mathbf{t}_{i}$, the state of context at time $i$, evolves according to the equation:

$$
\mathbf{t}_{i}=\rho_{i} \mathbf{t}_{i-1}+\beta \mathbf{t}_{i}^{\mathrm{IN}},
$$

where $\beta$ is a free parameter that determines the rate of contextual drift, $\rho_{i}$ is chosen at each time step such that $\mathbf{t}_{i}$ is always of unit length, and $\mathbf{t}_{i}^{\mathrm{IN}}$ is the input at time $i$. The property that context changes gradually with time allows TCM to predict recency effects for much the same reason as variable-context models (e.g., Mensink \& Raaijmakers, 1988) do. The input pattern $\mathbf{t}_{i}^{\mathrm{IN}}$, which will be described in more detail later, is context retrieved by item $\mathbf{f}_{i}$.

In TCM, each item is encoded in a specific temporal context. The current state of context cues items encoded in similar contextual states, as in Mensink and Raaijmakers's (1988) variable-context model of paired-associate learning. An outer-product matrix associates context vectors with item vectors:

$$
\mathbf{M}_{i}^{\mathrm{TF}}=\mathbf{M}_{i-1}^{\mathrm{TF}}+\mathbf{f}_{i} \mathbf{t}_{i-1}^{\prime},
$$

where the matrix $\mathbf{M}^{\mathrm{TF}}$ stores the association between an item and the previous state of context, indicated by the shift of index $i$. This index shift is not present in previously published treatments of TCM, but results in a more physical Hebbian association. Because $\mathbf{t}_{i}$ depends on the input caused by item $\mathbf{f}_{i}$, it is not present when $\mathbf{f}_{i}$ is first presented. In contrast, $\mathbf{t}_{i-1}$ is available at the time when $\mathbf{f}_{i}$ is presented. Given that Hebbian long-term potentiation is asymmetric (Levy \& Steward, 1983), introducing this change makes biophysical sense.

Contextual states serve as a cue for recall of items $\mathbf{f}$ via $\mathbf{M}^{\mathrm{TF}}$. Multiplying $\mathbf{M}^{\mathrm{TF}}$ by a context vector retrieves a superposition of the items $\mathbf{f}_{i}$, each activated to the extent that the probe context overlaps with the encoding context. If we probe with $\mathbf{t}_{j}$, the overlap is given by $\mathbf{t}_{i-1} \cdot \mathbf{t}_{j}$ :

$$
\mathbf{M}^{\mathrm{TF}} \mathbf{t}_{j}=\sum_{i}\left[\mathbf{f}_{i}\left(\mathbf{t}_{i-1} \cdot \mathbf{t}_{j}\right)\right] .
$$

Items that have been presented in a context similar to the probe context will be more strongly activated than those that have been presented in a completely dissimilar context.

Repeating an item at time step $r$, as a probe in a recall test or by means of recovering it in the process of free recall, results in an input pattern $\mathbf{t}_{r}^{\mathrm{IN}}$ that will overlap with other items' encoding contexts to a greater or lesser extent. Temporally defined associations in episodic memory have a characteristic form (Figure 1A). TCM accounts for this by assuming that multiple sources of information contribute to the context recovered by a cue item. Recall at time step $r$ of a list item previously studied at time $i$ will retrieve a combination of $\mathbf{t}_{i-1}$, the state of context that pre- 

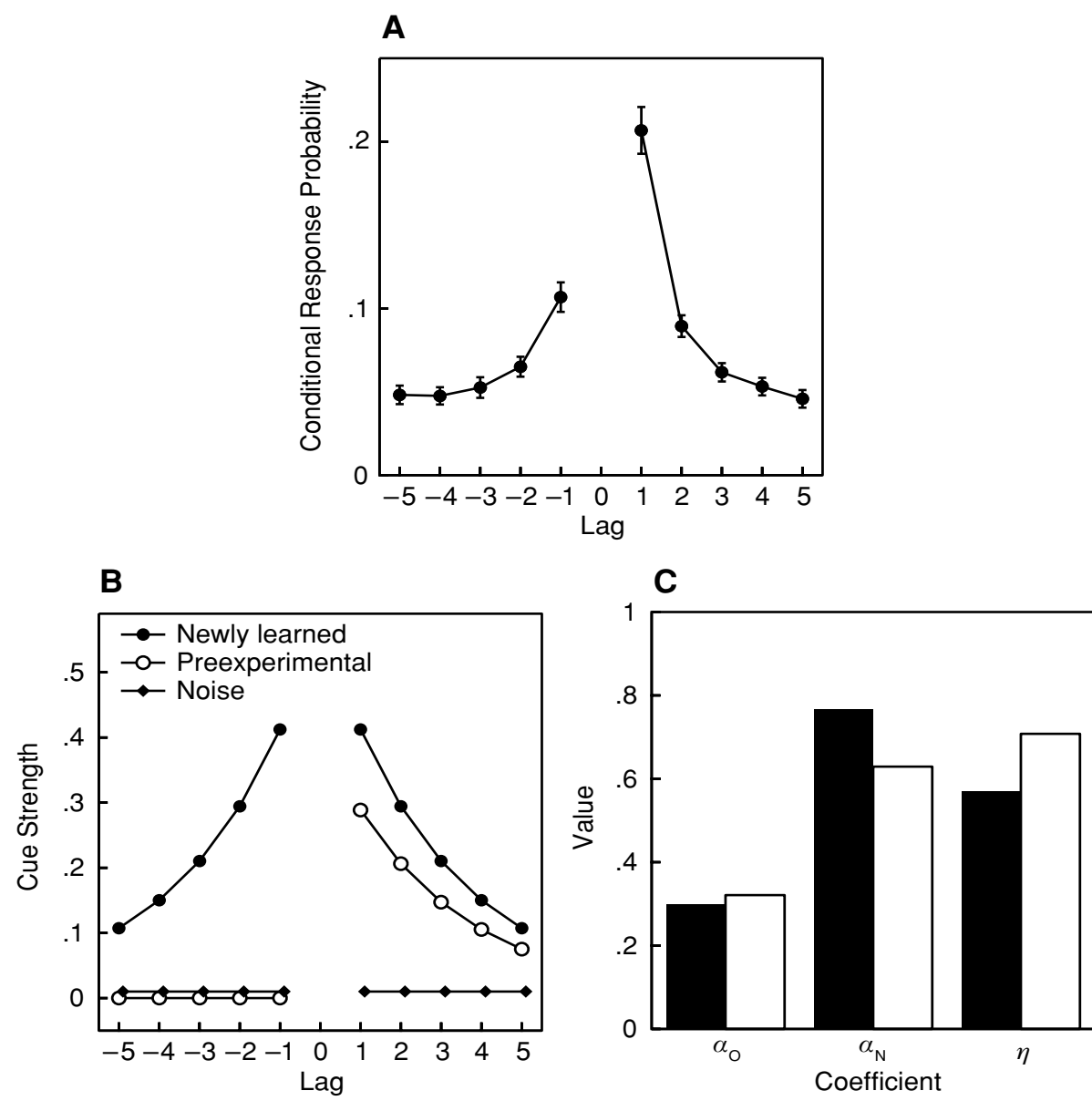

Figure 1. The lag recency effect. (A) Lag CRP function averaged across 11 free recall studies. The lag CRP measures the probability of recalling an item from position $i+$ lag immediately following an item from position $i$. The contiguity effect can be seen in the finding that lag CRP curves are peaked around zero. Asymmetry can be seen by noting that transitions in the forward direction (e.g., +1) are more likely than transitions in the backward direction (e.g., -1). Error bars are standard errors calculated across the experiments, which varied widely in their procedures. (B) The three components of retrieved context that combine to produce the lag CRP in TCM. The newly learned component, weighted by $\alpha_{N}$, provides a symmetric retrieval cue to the neighbors of the just recalled item (filled circles). The preexperimental component, weighted by $\alpha_{0}$, provides an asymmetric retrieval cue (open circles). A noise component, weighted by $\eta$, does not overlap with the study context of any of the list items (filled diamonds). The contribution of the noise component has been offset slightly for clarity. (C) Bestfitting parameter values for the three components for younger (filled bars) and older (open bars) adults. Older adults showed increased noise, consistent with disrupted associations. However, rather than being distributed evenly across the two beneficial components, the fits to older adults data showed a decrease only in $\alpha_{\mathrm{N}}$, accompanied by a small increase in $\alpha_{\mathrm{O}}$.

ceded the item when it was presented, the preexperimental context, $\mathbf{t}_{i}^{\mathrm{IN}}$, that item $i$ retrieved when it was presented, and $\mathbf{n}_{r}$, a noise component present at retrieval:

$$
\mathbf{t}_{r}^{\mathrm{IN}}=\alpha_{\mathrm{O}} \mathbf{t}_{i}^{\mathrm{IN}}+\alpha_{\mathrm{N}} \mathbf{t}_{i-1}+\eta \mathbf{n}_{r}
$$

The three factors in Equation 4 have different associative properties. The first two terms are effective cues for nearby items to the extent that the retrieved context overlaps with the items' encoding context (Equation 2). The cue strength to item $j$ from $\mathbf{t}_{i-1}$ is given by $\mathbf{t}_{i-1} \cdot \mathbf{t}_{j-1}$. Because the dot product is symmetric, $\mathbf{t}_{i-1}$ provides a sym- metric retrieval cue for the neighbors of item $i$ (Figure 1B, filled circles). The ability to recover $\mathbf{t}_{i-1}$ when item $i$ is repeated must mean that at study the representation of the item in semantic memory is associated to the current temporal context; $\mathbf{t}_{i-1}$ is referred to as newly learned context. The contribution of $\mathbf{t}_{i-1}$ is weighted by a coefficient $\alpha_{N}$. The other component of $\mathbf{t}_{r}^{\mathrm{IN}}$ that facilitates recall of nearby items is $\mathbf{t}_{i}^{\mathrm{IN}}$, the input pattern caused by the repeated item when it was originally presented. This input pattern persists as part of contextual states that follow item $i$ (Equation 1), but was not present before item $i$ was presented. 
This means that $\mathbf{t}_{i}^{\mathrm{IN}}$ provides an effective retrieval cue for items that followed the item $i$, not those that preceded it. The result is that $\mathbf{t}_{i}^{\mathrm{IN}}$ provides an asymmetric retrieval cue (Figure 1B, open circles). These first two components were included in previous treatments of TCM (Howard et al., 2005; Howard \& Kahana, 2002); they combine to provide an asymmetric lag CRP function with a gradient in the backward direction.

The third term in Equation 4 is a random noise vector that was not included in previous treatments of TCM. The dissociation between recency and lag recency observed for older adults (Kahana et al., 2002) could be described by traditional two-store models as a result of intact shortterm store and disrupted transfer to long-term store. Because contextual drift, which leads to the recency effect, and contextual retrieval, which leads to the lag recency effect, are so closely related in TCM (Equation 1), describing this dissociation is less straightforward. To decouple the rate of contextual and associative effects, we must fix the magnitude of $\mathbf{t}_{i}^{\mathrm{IN}}$ (Equation 4 ) across age groups while including a component of $\mathbf{t}_{r}^{\mathrm{IN}}$ that is not an effective retrieval cue. Because the random noise vector $\mathbf{n}_{r}$ is uncorrelated with the contextual states during list presentation, it will not provide an advantage for neighbors of item $i$. The noise term is weighted by a coefficient $\eta$.

Figure 1B summarizes the effect of each of these three components as a retrieval cue for items near $i$. The coefficients are manipulated subject to the constraint that $\mathbf{t}^{\mathrm{IN}}$ is always of unit magnitude. Across age groups, we allow the weighting on the retrieved noise, $\eta$, and the ratio of the two retrieved context components, $\alpha_{\mathrm{N}} / \alpha_{\mathrm{O}}$, to vary as free parameters.

\section{Modeling the Dissociation Between Recency and Lag Recency}

We fit the extended version of TCM to experimental data on recency and lag recency in young and older adults (Kahana et al., 2002). We simultaneously fit the PFR from immediate recall (Experiment 1 of Kahana et al., 2002) and the lag CRP from delayed recall (Experiment 2 of Kahana et al., 2002). Probability of recall was calculated from the model activations, using the Luce choice rule that has been used in previous implementations of TCM (e.g., Howard, 2004; Howard \& Kahana, 2002).

The Luce choice rule contributes a parameter, $\tau$, that controls the sensitivity of the retrieval process. We also included a parameter that increased the cue strength of the first list item to capture participants' tendency to occasionally begin recall with the first list item (the primacy effect, as seen in the PFR). This primacy parameter was allowed to vary separately for immediate and delayed free recall, although it was not allowed to vary across age groups. In all, five free parameters were varied: $\tau$; the primacy parameter; $\beta$, which controlled the rate of contextual drift (Equation 1); $\alpha_{\mathrm{N}} / \alpha_{\mathrm{O}}$, which determined the ratio of newly learned to preexperimental context in $\mathbf{t}_{r}^{\mathrm{IN}}$; and $\eta$, which controlled the amount of noise in $\mathbf{t}_{r}^{\mathrm{IN}}$ (Equation 4). The primacy parameter, $\beta$, and $\tau$ were fixed across age groups; the other two parameters, $\alpha_{\mathrm{N}} / \alpha_{\mathrm{O}}$ and $\eta$, were allowed to vary separately for each age group. A downhill simplex algorithm was used to search for the parameter set that minimized the $\chi^{2}$ between observed and predicted values.

Figure 2 shows PFR and lag CRP functions for young and older participants, along with the best fit of TCM to these data. One can see that young and older adults' PFR functions are nearly identical in both immediate and delayed free recall (Figure 2A). As can be seen by comparing Figures $2 \mathrm{~A}$ and $2 \mathrm{~B}, \mathrm{TCM}$ captures the three salient features of the PFR functions: (1) the pronounced recency effect in immediate free recall, (2) the elimination of recency in delayed free recall, and (3) the close correspondence of both these functions across age groups. The inclusion of a free parameter to describe the primacy effect has, not surprisingly, resulted in a primacy effect in the model fits that parallels the large primacy effect in delayed free recall and the smaller primacy effect in immediate free recall.

TCM was also successful in simultaneously fitting the age-related associative deficit in episodic recall (Figure $2 \mathrm{C}$ ). The fit to the lag CRP functions was statistically acceptable $\left[\chi^{2}(12)=18.0, p>.1\right]$ for young and older adults. Not surprisingly, the model settled on a fit in which the noise parameter was larger for older adults than for younger adults (Figure 1C). Interestingly, however, the best-fitting parameters did not result in a comparable decrease in the values of $\alpha_{\mathrm{O}}$ and $\alpha_{\mathrm{N}}$ for older adults. Rather, while $\alpha_{\mathrm{N}}$ decreased, the value of $\alpha_{\mathrm{O}}$ was actually larger for older adults than for younger adults. To determine whether this differential effect of aging on $\alpha_{\mathrm{O}}$ and $\alpha_{\mathrm{N}}$ was quantitatively reliable, we fit a control version of the model in which the $\alpha_{\mathrm{N}} / \alpha_{\mathrm{O}}$ was fixed across age groups. This control simulation did not provide an acceptable fit to the lag CRPs $\left[\chi^{2}(13)=54.3, p<.001\right]$. Moreover, the difference in fit between the models was significant $\left[\chi^{2}(1)=36.4\right.$, $p<.001]$. We conclude that aging had a differential effect on the model parameters such that the associative deficit observed in older adults was solely attributable to a decrement in $\alpha_{N}$.

\section{Discussion}

We extended TCM to account for the dissociation between recency and lag recency in the free recall of older adults. By allowing the magnitude of the components of retrieved context to vary, TCM provided excellent fits to both the young and older adults' lag CRP curves. Manipulating the noise parameter had a dramatic effect on lag recency while leaving the recency effect unaffected. The fits argue that the effect of normal aging in these data is to lower the value of $\alpha_{N}$, which weights the degree of new item-tocontext binding. This component of retrieved context has been hypothesized to depend on hippocampal function (Howard et al., 2005). Although this is not the first article to attribute the mnemonic deficit with aging to changes in contextual encoding (see, e.g., Balota, Duchek, \& Paullin, 1989), the present modeling points to an extremely specific and subtle disruption in contextual retrieval. 
A

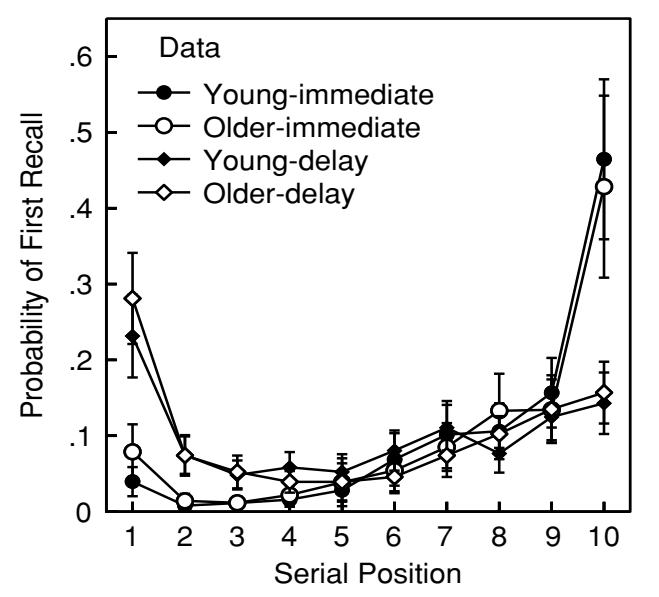

B

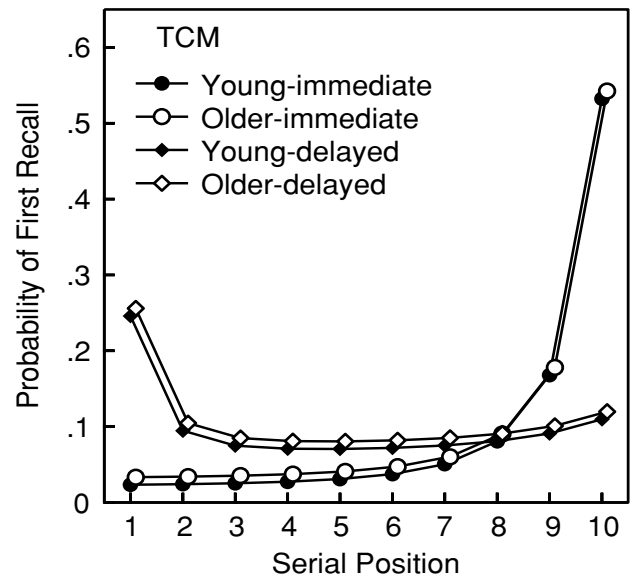

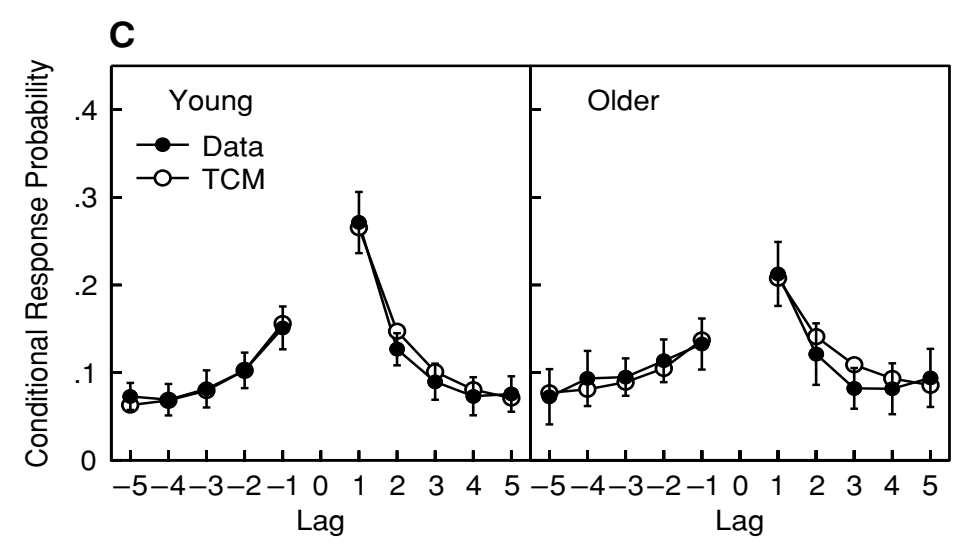

Figure 2. Recency and lag recency in normal aging. (A) Probability of first recall (PFR) functions for young and older adults in immediate and delayed free recall (Experiments 1 and 2 of Kahana et al., 2002). (B) Predicted PFR functions for young and older adults in immediate delayed free recall (fits to the data in A). In this panel, the older adults' data have been shifted slightly for clarity. (C) Observed and predicted lag CRP functions for young and older adults in delayed free recall. Error bars are $95 \%$ confidence intervals. Best-fitting parameter values were $\beta=0.77$ and $\tau=0.40$ (see also Figure 1C).

The finding of reduced item-context binding in older adults observed here, coupled with the ample evidence for disruptions in hippocampal physiology with aging (Rosenzweig \& Barnes, 2003), suggests that the hippocampus does play an essential role in binding items to a representation of temporal context (Howard et al., 2005). The present results can be seen as one possible implementation of the associative deficit hypothesis of normal aging (NavehBenjamin, 2000). Recently Li, Naveh-Benjamin, and Lindenberger (2005) have hypothesized that the associative deficit is a consequence of reduced input-output gain in older adults. Li et al. suggested that their manipulation of the gain parameter could be a proxy for changes in dopaminergic function in the prefrontal cortex with aging. Although a discussion of the relationship between prefrontal and medial temporal physiology is outside the scope of this article, we simply note here that the frontal cortex and hippocampus may be closely linked physiologically
(Hyman, Zilli, Paley, \& Hasselmo, 2005; Siapas, Lubenov, $\&$ Wilson, 2005); as a consequence, these hypotheses are not necessarily mutually exclusive.

Although it provides a principled account of lag recency effects, the present version of TCM is unable to describe dissociations between immediate recency (IR) and longterm recency (LTR) effects. For instance, the latency to recall the first items is much faster in immediate than in continuous-distractor free recall (Howard \& Kahana, 2005). A number of other potentially important distinctions between IR and LTR can be identified, including changes in the lag CRP with output position in immediate recall (Howard \& Kahana, 1999), the insensitivity of immediate recency to proactive interference (Murdock, 1962), and the sensitivity of LTR to proactive interference (Davelaar, Goshen-Gottstein, Ashkenazi, \& Usher, 2005).

The differences between IR and LTR effects motivate the theoretical position that attributes IR to rapid recovery 
of information from STS and LTR to a gradually changing contextual cue, not unlike the temporal context vector in TCM. Davelaar et al. (2005) have proposed such a model, in which IR reflected retrieval from STS and LTR reflected context-mediated retrieval from LTS. Whereas previous two-store models (Kahana, 1996; Raaijmakers $\&$ Shiffrin, 1980) described lag recency as a consequence of interitem associations formed between items coactive in STS, Davelaar et al. used a time-varying context signal to model the lag recency effect.

TCM embodies the distinction between activity-based memory (corresponding to STS) and weight-based memory (corresponding to LTS) that is central to dual-store models of episodic memory. The current state of $\mathbf{t}_{i}$ is an activity-based memory. Input patterns are turned on when the corresponding item is presented. These input patterns then decay gradually as new information is presented. As these patterns decay, information is lost. However, unlike STS in traditional two-store models, the activity-based memory in TCM decays gradually rather than in an all-ornone fashion. The matrix $\mathbf{M}^{\mathrm{TF}}$ is a weight-based memory system that persists over time. TCM also has a built-in distinction between IR and LTR effects. Although the relative differences in the activation of list items is similar in immediate and continuous-distractor free recall, the absolute level of activation is very different across the conditions. Perhaps the Luce choice rule can be replaced by a more detailed model of the dynamics that lead to recall of an item, given a set of activations as described by TCM.

In summary, we have extended the temporal context model (Howard et al., 2005; Howard \& Kahana, 2002) to account for the dissociation between recency and lag recency observed with normal aging (Kahana et al., 2002). We found that the model was able to successfully account for the dissociation between recency and lag recency. Lag CRP curves from younger and older adults were well fit quantitatively by the model. The results of this modeling effort argue that normal aging has a differential effect on the components of contextual retrieval, such that normal aging is associated with a decrease in the ability of items to recover the temporal context in which they have been encoded, a function that has been hypothesized to depend on the hippocampus proper (Howard et al., 2005). The differential effect of aging validates the distinction between the two components of retrieved context postulated by TCM.

\section{REFERENCES}

Atkinson, R. C., \& Shiffrin, R. M. (1968). Human memory: A proposed system and its control processes. In K. W. Spence \& J. T. Spence (Eds.), The psychology of learning and motivation (Vol. 2, pp. 89105). New York: Academic Press.

Balota, D. A., DucheK, J. M., \& Paullin, R. (1989). Age-related differences in the impact of spacing, lag, and retention interval. Psychology \& Aging, 4, 3-9.

Brodie, D. A., \& Murdock, B. B. (1977). Effects of presentation time on nominal and functional serial position curves in free recall. Journal of Verbal Learning \& Verbal Behavior, 16, 185-200.
Davelaar, E. J., Goshen-Gottstein, Y., Ashrenazi, A., \& Usher, M. (2005). A context activation model of list memory: Dissociating shortterm from long-term recency effects. Psychological Review, 112, 342 .

Glenberg, A. M., \& Swanson, N. G. (1986). A temporal distinctiveness theory of recency and modality effects. Journal of Experimental Psychology: Learning, Memory, \& Cognition, 12, 3-15.

Head, D., Buckner, R. L., Shimony, J. S., Williams, L. E., AkbuDAK, E., CONTURo, T. E., ET AL. (2004). Differential vulnerability of anterior white matter in nondemented aging with minimal acceleration in dementia of the Alzheimer type: Evidence from diffusion tensor imaging. Cerebral Cortex, 14, 410-423.

Hogan, R. M. (1975). Interitem encoding and directed search in free recall. Memory \& Cognition, 3, 197-209.

Howard, M. W. (2004). Scaling behavior in the temporal context model. Journal of Mathematical Psychology, 48, 230-238.

Howard, M. W., Fotedar, M. S., Datey, A. V., \& Hasselmo, M. E. (2005). The temporal context model in spatial navigation and relational learning: Toward a common explanation of medial temporal lobe function across domains. Psychological Review, 112, 75-116.

HowARD, M. W., \& KaHANA, M. J. (1999). Contextual variability and serial position effects in free recall. Journal of Experimental Psychology: Learning, Memory, \& Cognition, 25, 923-941.

HowARD, M. W., \& KAHANA, M. J. (2002). A distributed representation of temporal context. Journal of Mathematical Psychology, 46, 269-299.

Howard, M.W., \& Kahana, M. J. (2005). [Unpublished observation]. Hyman, J. M., Zilli, E. A., Paley, A. M., \& Hasselmo, M. E. (2005). Medial prefrontal cortex cells show dynamic modulation with the hippocampal theta rhythm dependent on behavior. Hippocampus, 15, 739-749.

KahANA, M. J. (1996). Associative retrieval processes in free recall. Memory \& Cognition, 24, 103-109.

Kahana, M. J., Howard, M. W., Zaromb, F., \& Wingfield, A. (2002). Age dissociates recency and lag-recency effects in free recall. Journal of Experimental Psychology: Learning, Memory, \& Cognition, 28, 530-540.

LAMING, D. (1999). Testing the idea of distinct storage mechanisms in memory. International Journal of Psychology, 34, 419-426.

LEVy, W. B., \& STEWARD, O. (1983). Temporal contiguity requirements for long-term associative potentiation/depression in the hippocampus. Neuroscience, 8, 791-797.

Li, S. C., Naveh-Benjamin, M., \& Lindenberger, U. (2005). Aging neuromodulation impairs associative binding. Psychological Science, 16, 445-450.

Mensink, G.-J. M., \& RaAiJmakers, J. G. W. (1988). A model for interference and forgetting. Psychological Review, 95, 434-455.

Murdock, B. B. (1962). The serial position effect of free recall. Journal of Experimental Psychology, 64, 482-488.

Murdock, B. B. (1997). Context and mediators in a theory of distributed associative memory (TODAM2). Psychological Review, 1997, 839-862.

Naveh-Benjamin, M. (2000). Adult age differences in memory performance: Tests of an associative deficit hypothesis. Journal of Experimental Psychology: Learning, Memory, \& Cognition, 26, 11701187.

Naveh-Benjamin, M., Hussain, Z., Guez, J., \& Bar-On, M. (2003). Adult age differences in episodic memory: Further support for an associative-deficit hypothesis. Journal of Experimental Psychology: Learning, Memory, \& Cognition, 29, 826-837.

RaAiJmakers, J. G. W., \& Shiffrin, R. M. (1980). SAM: A theory of probabilistic search of associative memory. In G. H. Bower (Ed.), The psychology of learning and motivation: Advances in research and theory (Vol. 14, pp. 207-262). New York: Academic Press.

Raz, N., Gunning, F. M., Head, D., Dupuis, J. H., McQuain, J., BRIGGS, S. D., ET AL. (1997). Selective aging of the human cerebral cortex observed in vivo: Differential vulnerability of the prefrontal gray matter. Cerebral Cortex, 7, 268-282. 
Rosenzweig, E. S., \& BARnes, C. A. (2003). Impact of aging on hippocampal function: Plasticity, network dynamics, and cognition. Progress in Neurobiology, 69, 143-179.

Rundus, D. (1971). An analysis of rehearsal processes in free recall. Journal of Experimental Psychology, 89, 63-77.

Siapas, A. G., Lubenov, E. V., \& Wilson, M. A. (2005). Prefrontal phase locking to hippocampal theta oscillations. Neuron, 46, 141151.
TAN, L., \& WARD, G. (2000). A recency-based account of the primacy effect in free recall. Journal of Experimental Psychology: Learning, Memory, \& Cognition, 26, 1589-1626.

WeST, R. L. (1996). An application of prefrontal cortex function theory to cognitive aging. Psychological Bulletin, 120, 272-292.

(Manuscript received April 23, 2003; revision accepted for publication July 8,2005 .) 